\title{
Bacteriological Profile of Neonatal Sepsis: A Hospital Based Study
}

\author{
Shrestha NJ'1, Subedi KU², Rai GK ${ }^{3}$
}

${ }^{1}$ Dr. Nisha Jyoti Shrestha, MBBS. MD, Registrar, ${ }^{2}$ Dr. Kalpana Upadhya Subedi, MBBS. DCH, MD, Associate Professor in Paediatrics (NAMS), ${ }^{3}$ Dr. Ganesh Kumar Rai, MBBS. DCH, MD. Associate Professor in Paediatrics (NAMS). All from the Department of Paediatrics, Kanti Children's Hospital, Maharajgunj, Kathmandu, Nepal.

Address for correspondence: Dr. Nisha Jyoti Shrestha, E-mail: nishajyoti@hotmail.com

\begin{abstract}
Introduction: Neonatal sepsis is a major cause of mortality and morbidity in newborn. There are many factors that contribute to neonatal sepsis. The organisms responsible for early onset and late onset sepsis are different. Objective: This study was conducted to analyze the organisms responsible for early onset and late onset neonatal sepsis. Materials and Methods: A prospective hospital based study over the period of one year was conducted at neonatal intermediate care unit of Kanti Children's Hospital, Maharajgunj, Kathmandu, Nepal. Results: Organisms were isolated in $6.1 \%$ of the collected blood samples. The male female ratio of culture proven sepsis was 1.9:1. Escherichia coli were found to be the most common organism in both early onset and late onset sepsis. Staphylococcus aureus was more common in late onset sepsis than early onset sepsis. Conclusion: Escherichia coli were the most common organism in both early onset and late onset sepsis. Staphylococcal aureus was significantly more common in late onset sepsis than early onset sepsis.
\end{abstract}

Key words: Bacteriological profile, early onset sepsis, late onset sepsis, neonate

\section{Introduction}

$\mathrm{N}^{\mathrm{e}}$ eonatal sepsis is one of the leading causes of hospital admission in Nepal and one of the leading causes of neonatal deaths ${ }^{1}$. Nepal has been working hard to achieve Millennium Development Goal (MDG) on reducing child mortality ${ }^{2}$. One of the components of this goal is reduction of infant mortality rate (IMR). The target IMR in Nepal by 2015 is 34 per 1000 live births. The trend of reduction of neonatal mortality is still not satisfactory. It is obvious that reduction of IMR is not possible without reducing neonatal morality rate $(\mathrm{NMR})^{1}$. It is estimated that $20 \%$ of all neonates develop sepsis ${ }^{3}$ and it is responsible for $30-50 \%$ of total neonatal deaths in developing countries. The reported incidence of neonatal sepsis varies from 7.1 to 38 per 1000 live births in Asia 5 . However; it is much lower in the developed countries. For instance, it ranges from 1.5 to 3.5 per 1000 for early onset sepsis (EOS) and up to 6 per 1000 live births for late onset sepsis (LOS) in USA and Australia, and it comprise a total of 6-9 per 1000 for neonatal sepsis ${ }^{5}$. Infection can occur either in fetal life, during birth or after birth. It can be acquired either from the community or at the nursery in the hospital.

There are many additional factors that predispose newborns in developing countries at a greater risk for developing neonatal sepsis compared with newborns in developed countries. These include intrinsic factors and extrinsic factors in the antenatal, intra-partum and the neonatal period ${ }^{6}$. Intrinsic factors in the developing world include higher rates of prematurity, intrauterine growth retrardation, birth asphyxia, premature and prolonged rupture of membranes and maternal peripartum infections. Among the most important extrinsic factors contributing to the high risk of sepsis are the lack of antenatal care, unhygienic birth practices and birth attended by an untrained birth attendant. The absence of skilled personnel at delivery also results in a failure to identify and refer high-risk newborns to better centers and a delay in managing complications when they occur. 
Neonatal sepsis is broadly divided according to age of onset into 2 types: early onset sepsis ( $<72 \mathrm{hrs})$ and late onset sepsis $(\geq 72 \mathrm{hrs}-28 \text { days })^{7}$. This classification is generally of importance for identification of predominant organisms causing infections during these phases. Early onset sepsis is acquired during fetal life, delivery or at the nursery and Group B streptococcus, Escherichia coli or Listeria monocytogens happen to be most common organisms ${ }^{8}$. Late onset sepsis is most commonly caused by Coagulase-negative staphylococci (CONS), Staphylococcus aureus, Escherichia coli, Klebsiella and Pseudomonas and is usually acquired in the neonatal intensive care unit (NICU) or the community ${ }^{9}$.

Periodic evaluation of organisms responsible for neonatal sepsis is essential for the appropriate management of neonates. Therefore, this study was conducted to find out the common organisms causing sepsis in neonates admitted in a tertiary level children's hospital.

\section{Methodology}

This was a prospective study conducted in the neonatal intermediate care unit (NIMCU) of kanti Children's Hospital (KCH) over one year period (September, 2008 to August, 2009) in neonates with suspected sepsis. There were a total of 1419 neonates admitted in the neonatal wards during this period. Among them blood culture was collected from 1349 neonates. The neonates were suspected having sepsis if they presented with one of the following signs or symptoms: fever (temperature $>38^{\circ} \mathrm{C}$ ), Hypothermia (temperature $<36^{\circ} \mathrm{C}$ ), decreased sucking, poor sucking or not sucking, lethargy, irritability, seizure, apnea, cough, respiratory distress, abdominal distention. Only those who had positive blood cultures were enrolled in this study. Blood samples were collected by aseptic vein puncture and were subjected to bacteriological culture. Blood sample collected for culture was incubated at $37^{\circ} \mathrm{C}$ over night and sub-culture on MacConkey agar was done. Growth negative culture was further incubated for another 3 days watching for growth every $24 \mathrm{hrs}$. Records of positive blood culture cases were entered in separate record file. Cases were classified as early onset sepsis if they presented at the hospital at less than $72 \mathrm{hrs}$ of life and late onset sepsis if at or after $72 \mathrm{hrs}$ of life. Data analysis was done using statistical package for social sciences (SPSS) software version 14.0. The level of significance for tests was set at $p<0.05$.

\section{Results}

During the study period there were 1419 neonates admitted in the intermediate ward. Among them blood cultures were done in 1349 cases as a part of septic screening in suspected neonatal sepsis cases. There were $859(63.7 \%)$ male and 490 (36.3\%) female neonates among them with the male to female ratio of 1.7:1. The total number of culture positive cases was found to be 82 with the culture positivity rate of $6.1 \%$. Among the culture positive cases there were 54 (65.9\%) males and $28(34.1 \%)$ female neonates with the male to female ratio of $1.9: 1$. The age of culture positive neonates ranged from 12 hours to 28 days with the mean age of 11.2 days. $E$ coli constituted the majority of the isolated organisms followed by Staph aureus. There were only a few cases of Klebsiella sp and Strep viridans (Table 1). Late onset sepsis cases were found to be five times higher than early onset sepsis (Fig. 1).

E coli were responsible in more than $3 / 4^{\text {th }}(76.9 \%)$ of the cases in early onset sepsis. It made only $49.2 \%$ of the cases followed by Staph aureus (44.9\%) in late onset sepsis (Fig. 2)

As shown in Table 2, E coli were isolated more in both early onset and late onset sepsis, however, Staph aureus was more common in late onset sepsis as compared with early onset sepsis and was statistically significant $(p=0.046)$.

Table 1: Distribution of isolated organisms $(n=82)$

\begin{tabular}{|l|c|c|}
\hline Organisms & Number & Percentage(\%) \\
\hline E coli & 44 & 53.7 \\
\hline Staph aureus & 33 & 40.2 \\
\hline Klebsiella sp & 3 & 3.7 \\
\hline Strept viridans & 2 & 2.4 \\
\hline Total & $\mathbf{8 2}$ & $\mathbf{1 0 0 . 0}$ \\
\hline
\end{tabular}

Table 2: Comparison of common isolated organisms by type of onset of sepsis $(n=77)$

\begin{tabular}{|l|c|c|c|c|}
\hline & EOS sepsis No (\%) & LOS sepsis No (\%) & Total cases (n) & $\boldsymbol{P}$ value \\
\hline E coli & $10(83.3)$ & $34(52.3)$ & 44 & $p=0.06$ \\
\hline Staph aureus & $2(16.7)$ & $31(46.7)$ & 33 & $p=0.046$ \\
\hline Total & $\mathbf{1 2}$ & $\mathbf{6 5}$ & $\mathbf{7 7}$ & - \\
\hline
\end{tabular}




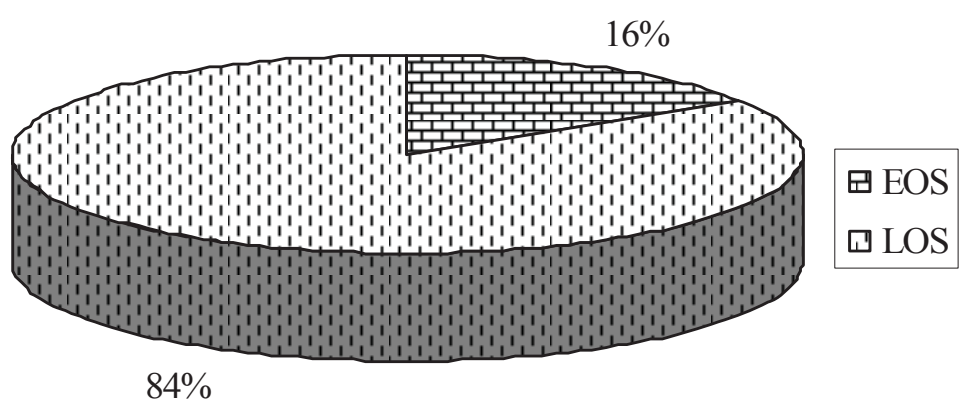

Fig 1: Distribution of cases by type of sepsis

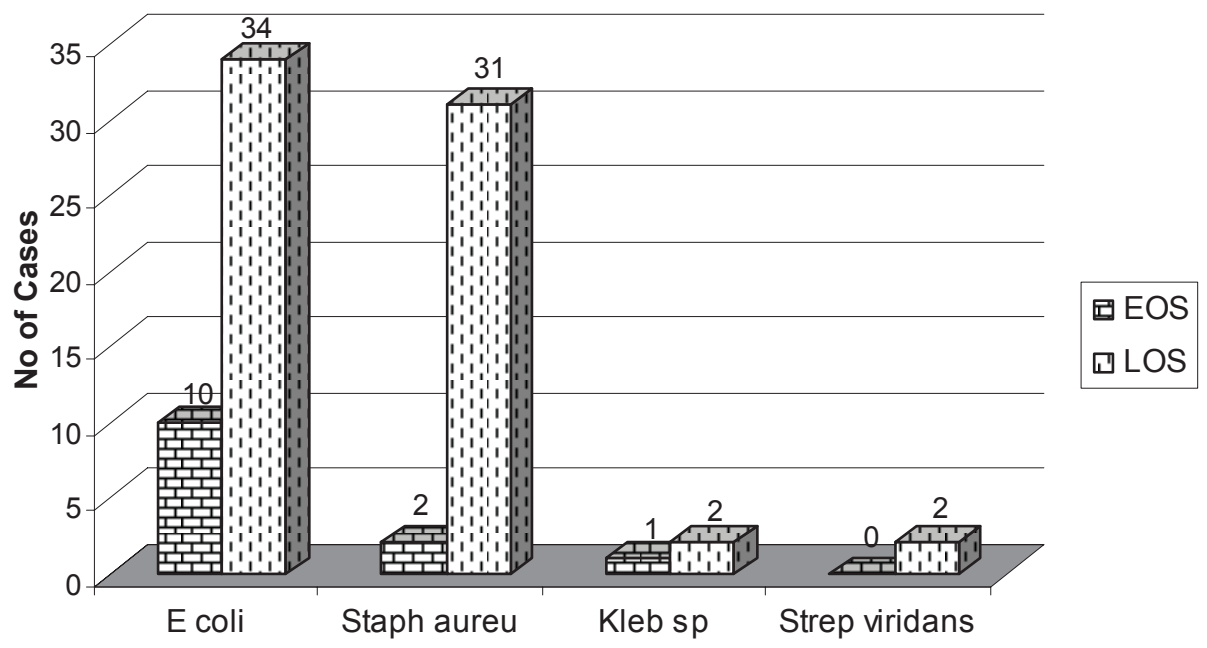

Fig 2: Distribution of pathogens in EOS \& LOS

\section{Discussion}

Neonatal sepsis is a leading cause of death among neonates in Nepal, other important causes being prematurity and low birth weight, birth asphyxia and hypothermia ${ }^{1}$. It is believed that appropriate management of neonates with sepsis can reduce neonatal morbidity and mortality and help to achieve Millennium Development Goals. Therefore, periodic evaluation and monitoring of causative organisms responsible for sepsis is of outmost importance in its management.

The culture positivity rate in this study was found to be $6.1 \%$, which is much lower as compared to other studies $\left(56.6 \%\right.$ by Jain NK et.al. ${ }^{10}, 21.0 \%$ by Barbara JS et. al. ${ }^{13}$ and $13.7 \%$ by Shrestha $S$ et. al. ${ }^{14}$ ). The low yield in our study could be due to the fact that this study was conducted in a tertiary care hospital where most of the cases are referred from other hospitals and health facilities. These babies could have already received antibiotics prior to referral.

This study has shown male female ratio of 1.9:1, which is similar to the findings by Jain NK et. al. $(2: 1)^{10}$ and by Jia-horng Jiang et. al. $(1.4: 1)^{11}$. Probably, this could be because of the priority given to male babies for medical care in our society.

It was revealed in this study that late onset sepsis constituted more than two third of the cases. This observation was in contrast with the finding by Jain NK et. $\mathrm{al}^{10}$. This is true for the hospital like ours that do not have birthing centre and most of the cases being referred from other health facilities a few days after birth.

$E$ coli pre-dominated the organisms isolated in sepsis cases in our study. This is in agreement with some other studies ${ }^{10,13,14}$. Jia-horng Jiang et. al. ${ }^{11}$ also reported that there was an increasing trend of prevalence of neonatal sepsis caused by gram negative organisms like $E$ coli. It is seen globally that the overall ratio of gram-negative organisms to gram-positive organisms is 2:1.6 However, a study conducted in South Korea by Youn-Jeong et al found $E$ coli only in $9.3 \%$ of the cases ${ }^{18}$. It is very surprising that not a single case of Group B Streptococcus (GBS) was isolated in our study.

In our study E coli constituted more than two thirds of the organisms in early onset sepsis. This was similar 
to findings by other studies (Vergnano et. al. ${ }^{5}$ Jia-horng Jiang et. al. ${ }^{11}$ and Barbara JS et. al. ${ }^{13}$ ). However, this was in contrast with the finding by Michael CohenWolkowiez et. al. ${ }^{16}$ who observed that gram-positive organisms constituted the majority of early onset sepsis. This result could be because of predominance of Group $B$ Streptococcus (GBS) in developed countries.

This study showed statistically significant predominance of Staph aureus in late onset sepsis as compared to early onset sepsis. Similar finding was also reported from India ${ }^{17}$. Coagulase negative staphylococcus (CONS) seemed to be more common in LOS sepsis than staphylococcus aureus ${ }^{11,12,14}$. However, a study conducted in eastern part of Nepal showed that both CONS and Staph aureus were the commonest organisms causing both EOS and LOS ${ }^{19}$.

\section{Conclusion}

In conclusion, $E$ coli were the commonest organism in both early onset and late onset sepsis. Staph aureus were significantly more common in late onset sepsis as compared to early onset sepsis.

\section{Acknowledgement}

I would like to express my sincere gratitude to all the medical officers of NIMCU, who helped to register the cases in log book. And I would like to thank Ms Maiya Thapa and Ms Gyani Singh of the Microbiology department of $\mathrm{KCH}$ for their support in this study.

\section{Funding: None \\ Conflict of Interest: None \\ Permission from IRB: Yes}

\section{References}

1. National neonatal health strategy. Family health division, Department of health services, Ministry of health, Government of Nepal, 2004; 1-4

2. Nepal MDGs progress reports, National planning commission, Government of Nepal, 2005; 37-42

3. Stoll BJ. Infections of the neonatal infant. In: Behrman RE, Kliegman RM, editors. Nelson textbook of pediatrics, $18^{\text {th }}$ edition. W.B. Saunders Company, 2008, p.794-98

4. Agrawal R, Sarkar N, Deorary AK and Paul VK. Sepsis in newborn. Indian J Paediatr 2001;68:114347.

5. Vergnano S, Sharland M, Kazembe P, Mwansambo C, Heath PT. Neonatal sepsis: an international perspective. Arch Dis Child Fetal Neonatal 2005;90:F220-24.

6. Ganatra HA, Stoll BJ, Zaidi AKM. International Perspective on early-onset neonatal sepsis. Clin Perinatol 2010;37:501-23

7. Puopolo KM. Bacterial and fungal infection. In: Cloherty JP, Eichenwald EC, Stark AR, editors. Manual of neonatal care, 6th edition. Wolters Kluwer/Lippincott William \& Wilkins, 2008, p. 274300

8. Klinger G, Levy I, Sirota L, Boyko V, Reichman B, Lerner-Geva L. Epidemiology and risk factors for early onset sepsis among very-low-birthweight infants. Am J Obstet Gynecol 2009;201:38.1-6.

9. Van den Hoogen A, Gerards LJ, VerboonMaciolek MA, Fleer A, Krediet TG. Longterm trends in the epidemiology of neonatal sepsis and antibiotic susceptibility of causative agents. Neonatology 2009;97:22-28.

10. Jain NK, Jain VM, Maheshwari S. Clinical profile of neonatal sepsis. Kathmandu Univ Med J 2003;1(2): $117-20$

11. Jiang JH, Chui NC, Huang FY, Kao HA, Hsu CH, Hung $\mathrm{HY}$ et al. Neonatal sepsis in the Neonatal intensive care unit: characteristics of early versus late onset. J Microbial Immunol Infect 2004;37:30106.

12. Stoll BJ, Hansen N, Fanaroff AA, Wright LL, Carlo WA, Ehrenkranz RA et al. Late-onset sepsis in very low birth weight neonates: The experience of the NICHD neonatal research network. Pediatrics 2002;110;285-91

13. Stoll BJ, Hansen N, Fanaroff AA, Wright LL, Carlo WA, Ehrenkranz RA et al. Changes in pathogens causing early onset sepsis in very low birth weight infants. N Engl J Med 2002; 25:240-47

14. Shrestha S, Adhikari N, Shakya D, Manandhar L, Chand A, Shah S. Bacteriological profile of neonatal blood cultures at Patan hospital. J. Nepal Paediatr. Soc 2008;26:9-12.

15. Sharma PP, Holder D, Dutta A. Bacteriological profile of Neonatal septicemia. Indian Paediatr 1987;11:1010-17.

16. Wolkowiez MC, Moran C, Benjamin DK, Cotton $\mathrm{CM}$, Clark RH, Benjamin DK, Jr. Early and late onset sepsis in late preterm infants. Pediatr Infect Dis J 2009;28:1052-56. 
17. Karthikeyan $\mathrm{G}$ and Premkumar K. Neonatal sepsis: Stapphylococcus aureus as the predominant pathogen. Ind J Pediatr 2001;68:715-17.

18. Youn-Jeong Shin, Ki M, Foxman B. Epidemiology of neonatal sepsis in South Korea. Pediatr International 2009;51:225-32
19. Shrestha P, Das BK, Bhatta NK, Jha DK, Das B, Setia A et al. Clinical and Bacteriological Profiles of Blood Culture Positive Sepsis in Newborns. J Nepal Paediatr. Soc. 2008; 27(2): 64-67.

\section{How to cite this article?}

Shrestha NJ, Subedi KU, Rai GK. Bacteriological Profile of Neonatal Sepsis: A Hospital Based Study. J Nepal Paedtr Soc 2011;31(1):1-5. 